\title{
Investigação e elaboração de uma proposta mobile learning para apoio ao ensino de enfermagem
}

\author{
Michelle Nayara Silva \\ Instituto Federal do Norte de Minas \\ Gerais \\ Januária, Brasil \\ michellenayara94@gmail.com
}

\author{
Felipe Augusto Oliveira Mota \\ Instituto Federal do Norte de \\ Minas Gerais \\ Januária, Brasil \\ felipe.mota@ifnmg.edu.br
}

\author{
Karine Alencar Fróes \\ Instituto Federal do Norte de Minas \\ Gerais \\ Januária, Brasil \\ karine.froes@ifnmg.edu.br
}

\begin{abstract}
The technological advance has more and more influence on the learning of health professionals, since the use of different technological resources such as Educational Software aims to facilitate the understanding and assimilation of the theoretical and practical content addressed in some procedures. In this context, the objective of this work was to carry out an investigation for the development of a solution based on mobile learning to support the teaching of nursing. The work was conducted at the Federal Institute of Northern Minas Gerais (IFNMG), Januária Campus. Initially, a questionnaire was applied with teachers to identify the contents that they have the most difficulty in teaching. After analysis, it was decided to work with the wounded theme. The next step was the development of a system proposal observing all the software engineering and usability requirements. The steps taken during the project open perspectives to believe that the use of this proposed application is of great effectiveness in the process of teaching nursing. As future work it is intended to develop the projected application.
\end{abstract}

Resumo- O avanço tecnológico tem cada vez mais influência sobre a aprendizagem dos profissionais da saúde, uma vez que a utilização de diferentes recursos tecnológicos como Softwares Educacionais visa facilitar o entendimento e a assimilação do conteúdo teórico e prático abordados em alguns procedimentos. Nesse contexto, objetivou-se neste trabalho realizar uma investigação para o desenvolvimento de uma solução baseada em mobile learning para o apoio ao ensino de enfermagem. $O$ trabalho foi conduzido no Instituto Federal do Norte de Minas Gerais (IFNMG), Campus Januária. Inicialmente realizou-se a aplicação de questionário com professores para identificar os conteúdos que eles têm mais dificuldade de ministração. Após análise, optou-se por trabalhar com o tema feridas. A próxima etapa foi o desenvolvimento de uma proposta de sistemas observando todas as exigências de engenharia de software e usabilidade. As etapas percorridas durante o projeto abrem perspectivas para acreditar que o uso desse aplicativo proposto é de grande eficácia no processo de ensino em enfermagem. Como trabalho futuro pretende-se desenvolver a aplicação projetada.

Palavras-chave - ensino; mobile learning; software educacional.

\section{INTRODUÇ̃̃O}

O profissional de enfermagem deve abranger um saber teórico e prático sobre determinados números de habilidades e conhecimentos da área da saúde. Desta maneira, [1] ressalta que o professor enfermeiro é o elemento fundamental no processo de ensino de um curso de enfermagem, é ele que possibilita a inserção de novos perfis profissionais qualificados e habilitados para exercerem esta profissão.

Dentre os inúmeros desafios na formação de profissionais de enfermagem, [2] destaca a dificuldade em realizar a relação teoria e prática; falta de material didático, audiovisual, laboratório e campo de estágio adequado, assim como a falta de tempo para ministrar o conteúdo proposto de modo que proporcione aulas mais atrativas e dinâmicas.

Conforme [3], o Instituto Federal do Norte de Minas Gerais (IFNMG) - Campus Januária é uma instituição de formação profissional que oferta cursos de nível médiotécnico, superior e de pós-graduação. Dentre os cursos ofertados pela Instituição tem-se o Curso Técnico em Enfermagem, com duração de dois anos, divididos em quatro períodos que visa: Desenvolver a capacidade de enfrentar situações e acontecimentos próprios do campo profissional, com autonomia, discernimento, iniciativa e responsabilidade, baseada em conhecimentos técnicos científicos e com inteligência prática para, de forma humanizada, avançar em direção à autonomia dos sujeitos em relação à própria saúde e a responsabilização coletiva pela promoção da saúde de indivíduos, grupos e meio ambiente.

No referido curso, existe uma grande preocupação com o ensino e consequentemente com aprendizado referente aos diversos conteúdos apresentados ao longo do curso, bem como das técnicas para atendimento ao público, uma vez que esse atendimento influencia diretamente na saúde da população.

As disciplinas Fundamentos de Enfermagem I, II e III contemplam as temáticas relacionadas a prática de enfermagem no atendimento ao paciente, desse modo no que se refere ao ensino desses conteúdos, quando se deseja que estudantes alcancem objetivos cognitivos mais elevados, como a compreensão e aplicação de determinados conhecimentos, a aula expositiva pode mostrar-se limitada. Para [4], nesses casos, os professores podem valer-se de recur- 
sos tecnológicos. Além disso, para alcançar a compreensão e a aplicação de certos conteúdos, recomenda-se a realização de experiências práticas. No entanto, a realização dessas experiências nem sempre é fácil. Algumas não podem ser reproduzidas em sala de aula. Assim os recursos tecnológicos, como Softwares Educacionais, tornam-se importantes estratégias à medida que possibilitam aos docentes superar as barreiras para a realização das experiências práticas, permitindo ao estudante uma aproximação com a realidade.

O ensino vem sofrendo muitas mudanças ao longo dos anos. Propostas pedagógicas defendidas por teóricos vão sendo questionadas quanto a sua eficiência e eficácia no aprendizado. Novas concepções pedagógicas têm se construído a partir do uso dos novos recursos tecnológicos. Incentivando o aluno a buscar informações, a aprender e questionar ampliando assim o seu aprendizado. De acordo com [5], a aprendizagem dos profissionais da saúde sofre cada vez mais influência do avanço tecnológico, uma vez que esses recursos visam facilitar o entendimento e a assimilação do conteúdo teórico e prático abordados em alguns procedimentos.

Assim, enfermeiro, enquanto docente, deve desenvolver estratégias de ensino que facilitem a aprendizagem. A utilização de diferentes recursos tecnológicos torna-se importante no processo de (re)construção dos saberes na enfermagem, visto que estas proporcionam o aprendizado a partir das múltiplas potencialidades, capacidades e interesses dos discentes.

Em complemento, [6] afirma que a introdução das novas Tecnologias da Informação e Comunicação (TIC) tem provocado mudanças na forma de acesso à informação, devido sua rápida evolução tecnológica, o que vem contribuindo para inserção de novas estratégias de ensino através do uso de uma diversidade de recursos que favorecem a melhoria do processo dele.

Sendo assim, é de extrema relevância que docentes de enfermagem desenvolvam e utilizem recursos educacionais, como Software Educacional como uma alternativa viável de ensino que pode proporcionar uma maior facilidade de consulta aos conteúdos relacionados, como, por exemplo, ao tema feridas e, dessa forma, ajudar no entendimento teórico e prático do conteúdo.

De acordo com [5], nas últimas décadas, como tecnologia adotada no ensino de enfermagem, tem se observado o aumento das tecnologias educacionais digitais (TED), entendidas como recursos informatizados utilizados no apoio ao ensino (vídeos, ambientes virtuais, softwares educacionais).

$\mathrm{Na}$ visão de [6], a progressão tecnológica nos ambientes de cuidado à saúde instigou uma melhora na educação. Tal modificação incentivou a introdução de tecnologias de ensino em enfermagem, que tentam conciliar conhecimentos teóricos, habilidades práticas e atitudes para a execução de procedimentos a serem desempenhadas.

Para [7], dentre os diversos recursos utilizados como meio de apoiar o ensino e a aprendizagem, atualmente contamos com os dispositivos móveis, tais como tablets e smartphones, a fim de permitir o acesso ao conhecimento em qualquer hora e em qualquer lugar, garantindo com que o aluno rompa as barreiras de tempo e espaço proporcionando a mobilidade durante sua utilização.

Conforme [8], o uso desses dispositivos móveis para o ensino e aprendizagem se caracteriza como mobile learning (m-learning), que se refere a uma terminologia evoluída do Eletronic Learning (e-learning).

$\mathrm{O}$ termo $m$-learning é a fusão de diversas tecnologias de processamento e comunicação de dados, que permite aos discentes e docentes uma maior interação relacionada ao processo de ensino aprendizagem, e a mobilidade dos mesmos.[9] Assim, o objetivo do presente trabalho é apresentar uma investigação com docentes acerca das dificuldades na ministração de conteúdo em um curso técnico em enfermagem, identificar um foco e, por fim, propor uma solução tecnológica para apoio ao ensino baseada em mobile learning.

\section{MOBILE LEARNING}

Segundo [8], as características dessa tecnologia permitem a construção do conhecimento de forma flexível utilizando dispositivos móveis além de ser mais leve do que um Notebook e permite ao usuário acessar informações por meio de texto, imagem, vídeo ou voz. Além de facilitar aos aprendizes a construção de seu próprio conhecimento; expandir os limites internos e externos da sala de aula; fornece meios para o desenvolvimento de métodos inovadores de ensino utilizando os novos recursos de computação.

Em sua análise [10] evidencia que o uso da m-leanirng tem contribuição positiva nas seguintes áreas:

- Melhora habilidades de alfabetização e numeramento;

- Aprendizagem independente e colaborativa, baseada em experiências;

- Ajuda alunos a identificar áreas onde precisam de ajuda e apoio;

- Ajuda a combater a resistência ao uso de TIC;

- Ajuda a remover algumas das formalidades da experiência de aprendizagem e auxilia no engajamento de alunos relutantes;

- Ajuda os alunos a permaneceram mais focados por períodos mais longos;

- Proporciona o aumento da autoestima dos alunos;

- Aumenta a autoconfiança do aluno.

O acesso fácil aos smartphones é outro ponto importante de ser destacado. Em sua pesquisa, [11] destaca que os smartphones são muito populares entre os alunos devido ao fato de oferecerem experiências de simplicidade e mobilidade no acesso a materiais de ensino e aprendizagem. Porém faz uma alerta quanto os desafios para combater a distração de manter o foco nas atividades de aprendizagem. Assim, sugere a utilização de materiais de multimidia além dos conteúdos textuais.

Segundo [12], a mobile learning deve ser constituída observando cinco pontos básicos: flexibilidade, colaboração, motivação, acessibilidade e portabilidade. A flexibilidade define que a aprendizagem pode acontecer em qualquer hora e em qualquer lugar. Já a colaboração significa que todos usam o mesmo conteúdo e isso poderá levar ao 
recebimento de feedback instantâneo e dicas. A colaboração também indica que é possível reduzir barreiras culturais e de comunicação entre professores e alunos.

A motivação é relacionada com as formas diferentes de aprendizagem, pois os alunos podem, por exemplo, aprender através de jogos, o que torna o estudo mais atrativo. A acessibilidade e portabilidade são diretamente associadas ao smartphone, já que ele permite um acesso rápido e virtual a diversos tipos de aplicações em qualquer lugar e por ser de pequeno porte e leve, pode ser levado para qualquer lugar.

Isso pode demonstrar uma tendência para o desenvolvimento tecnológico e a expansão de alternativas de aprendizagem utilizando dispositivos móveis. Em seu trabalho, [13] destaca que o m-learning crescerá extremamente rápido nos próximos anos, incentivado pela variedade dos dispositivos de aprendizagem móveis, com tecnologia cada vez mais poderosa e mais portáteis devido a autônima energética. Ainda acrescenta que os avanços tecnológicos nos smartphones, auxilia que os problemas relacionados a dispositivos sejam resolvidos rapidamente.

\section{MATERIAIS E MÉTODOS}

Em busca da contemplação do objetivo, realizou-se uma investigação de levantamento de dados através da aplicação de questionários (em anexo) que visava identificar quais conteúdos presentes nas disciplinas Fundamentos de Enfermagem I, II e III apesentavam maior limitação na prática de ensinar. Os dados foram coletados juntamente com professores do curso técnico de enfermagem do IFNMG - Campus Januária.

A tabulação e análise dos dados coletados ocorreu por intermédio de uma planilha eletrônica, cujos resultados foram organizados usando estatística descritiva simples. Segundo [14], esse tipo de estáticas correspondem a questões diretas cujas respostas se limitam a informações objetivas. Assim, foi possível quantificar, em forma de porcentagem, os dados obtidos. Ao fim da análise, foi definido um dos conteúdos para o desenvolvimento da aplacação, seguindo critérios pedagógicos e tecnológicos.

Após identificar o conteúdo para o desenvolvimento do aplicativo realizou-se uma segunda investigação a partir de questionário e entrevistas com os docentes para levantar quais pontos seriam importantes abordar no sistema em busca de atender as necessidades. Segundo [15], a importância de um levantamento de dados para o desenvolvimento de uma aplicação móvel pode auxiliar a melhor compreensão da problemática e reais necessidades dos potenciais usuários além de proporcionar uma visão de uma boa interface propicia ao usuário que seja eficaz e de fácil utilização.

Posteriormente efetuou-se a elaboração e as especificações do Software, definindo quais funcionalidades ele deve conter e como deve operar. Em seguida iniciou-se o desenvolvimento de um protótipo de software baseado nos requisitos elaborados. Conforme explica [16], um protótipo é uma amostra de um produto final rapidamente desenvolvida que demonstra algumas ou todas as suas capacidades funcionais.

O protótipo será elaborado observando os aspectos de interação humano computador (IHC). [17] define heurísticas que como intuito auxiliar os desenvolvedores de sistemas a construírem suas interfaces considerando os princípios de usabilidade.

Entretanto como específica [18] medir a usabilidade de uma interface envolve não apenas medir questões relativas às funcionalidades de um software, mas também a facilidade de seu uso como ferramenta de trabalho, tendo como um dos principais desafios a redução do tempo necessário para aprendermos a utilizar o sistema.

\section{DESENVOLVIMENTO DA INVESTIGAÇÃO}

No processo de coleta dos dados, foram entrevistados todos os professores do curso técnico de enfermagem do IFNMG - Campus Januária que compõe uma equipe de 10 docentes. Foi questionado aos professores se o desenvolvimento de um aplicativo contendo atividades interativas como jogos, animações e vídeos poderia auxiliar na prática de ensino dos conteúdos ministrados nas disciplinas Fundamentos I, II e III. O gráfico 1 a seguir aponta que todos os professores concordaram em relação a utilização de tecnologias educacionais conforme a questão apresentada.

TABELA I

\begin{tabular}{|c|c|}
\hline Opinião sobre o uso de aplicativo & Quantidade \\
\hline SIM & 10 \\
\hline NÃO & 0 \\
\hline TALVEZ & 0 \\
\hline tabela 1. Fonte: própria autora
\end{tabular}

Posteriormente realizou-se o questionamento aos docentes a respeito de quais conteúdos presentes nas disciplinas Fundamentos de Enfermagem I, II e III apresentava maior limitação na prática de ensinar. A tabela 1 apresenta os resultados dos questionários aplicado aos docentes. TABELA II

\begin{tabular}{|c|c|c|}
\hline Conteúdos & Quantidade & \% \\
\hline Nebulização / inalação & 0 & $0 \%$ \\
\hline Oxigenioterapia & 2 & $9 \%$ \\
\hline Punção venosa & 1 & $4 \%$ \\
\hline $\begin{array}{c}\text { Administração de me- } \\
\text { dicamentos }\end{array}$ & 2 & $9 \%$ \\
\hline Enema / enteróclise & 2 & $9 \%$ \\
\hline Cálculo de medicação & 9 & $41 \%$ \\
\hline Tratamento de feridas & 5 & $23 \%$ \\
\hline Retirada de pontos & 1 & $5 \%$ \\
\hline tabela 2. & Fonte: própria autora \\
\hline
\end{tabular}

Diante desses resultados os conteúdos que obtiveram maiores destaques foram "cálculo de medicação" e "tratamentos de feridas", em decorrência disso a equipe de pesquisa juntamente com a coordenação de curso e o acompanhamento pedagógico analisou sobre os temas e sua relevância em relação a enfermagem, como também a viabilidade referente ao desenvolvimento da aplicação mobile.

Então, discutiu-se sobre o tema "cálculo de medicação", que está relacionado diretamente à cálculos 
matemáticos, raciocínio lógico e conhecimento de fórmulas específicas. Dessa forma contatou-se que seria um tema muito extenso e complexo para abordar no aplicativo.

Por outro lado, o tema "tratamentos de feridas" traz conceitos e teorias que possibilita explorar diversos recursos pedagógicos.

Diante disso foram considerados os princípios do processo de ensino e da busca de torna-lo mais didático e intuitivo, o que resultou na comparação dos dois temas abordando a complexidade e as dificuldades de cada um, e concluiu-se que o conteúdo "tratamentos de feridas" seria um tema mais flexível e proveitoso de ser trabalhado podendo ser explorado os recursos interativos como visuais e auditivos, o que aumenta as chances de apresentar resultados satisfatórios.

O conteúdo de feridas é ministrado ao longo da disciplina Fundamentos de Enfermagem II, no segundo período. Durante as aulas da referida disciplina, são ensinados conteúdo do tema feridas, desde o conceito, formas de tratamento, tipos de coberturas, além da técnica de execução de curativo. Os alunos praticam os procedimentos em bonecos simuladores, sendo constantemente observados pelo docente, a fim de identificar e sanar suas dúvidas.

De acordo com [19], uma ferida como "qualquer interrupção da continuidade de um tecido corpóreo, em maior ou menor extensão, ocasionada por qualquer tipo de trauma físico, químico, mecânico ou desencadeada por uma afecção clínica". [20] afirma que o tratamento de feridas é uma atividade cotidiana do enfermeiro e profissionais da área da saúde em geral. A prática de cuidados a pacientes portadores de feridas é uma especialidade dentro da enfermagem, reconhecida pela Sociedade Brasileira de Enfermagem Dermatológica (SOBEND) e Associação Brasileira de Estomaterapia (SOBEST) e que requer conhecimento específico, habilidade e abordagem abrangente.

Segundo [21], o tratamento de feridas é um processo complexo e dinâmico, que depende de avaliações sistematizadas, prescrições distintas de frequência e tipos de curativos ou coberturas necessárias, de acordo com cada momento da evolução do processo cicatricial. A avaliação do profissional de enfermagem é de extrema importância, pois será ela que traçará a metodologia do tratamento para o portador de uma ferida.

No momento que foi determinado o tema "feridas" realizou-se uma entrevista com os professores que administram esse conteúdo, com a finalidade deles apresentarem quais os pontos que consideram mais relevantes dentro do conteúdo relacionado a feridas e quais assuntos possuía maior dificuldade para ensinar aos discentes. Dentre as dificuldades constatadas destacam-se:

- Classificação das feridas

- Tipos de coberturas

- Diferenciar os tipos de tecidos e secreções das feridas devido ausência de materiais visuais de qualidade

- Processo de cicatrização, características dos tecidos viáveis e inviáveis na lesão

- Técnica de realização de curativo conforme a classificação das feridas
- Técnica asséptica de manuseio das pinças, bem com o posicionamento da mesma no campo estéril

É importante ressaltar que os conteúdos listados necessitam de recursos visuais para facilitar o entendimento, uma vez que a principal dificuldade relatada pelos docentes foi a falta de materiais para ilustrar os conceitos que são ensinados em sala de aula.

\section{Proposta do Sistema}

A investigação anterior proporcionou a proposta do desenvolvimento do aplicativo móvel. Nesta etapa envolveuse o planejamento e a produção do conteúdo didático, a definição dos tópicos e produção da escrita dos assuntos, a seleção das mídias e o desenho da interface (layout).

$\mathrm{O}$ protótipo apresentado consiste um projeto inicial de trabalho ainda na fase de planejamento e definição de suas funcionalidades, que seguidamente dará início ao seu desenvolvimento a fim de produzir o resultado que foi especificado no projeto, bem como a realização de testes e a disponibilização o Software Educacional.

A proposta consiste que o usuário acesse o sistema e tenha disponibilidade de utilizar dois ambientes de estudo distintos, um ambiente de estudo textual e outro ambiente de estudo a partir de simulações como visto na figura 1 .

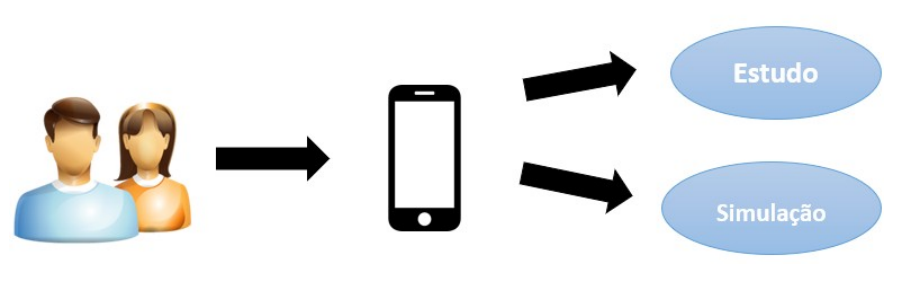

Fig. 1. Proposta geral do sistema proposto

Atentou-se para questões relativas à atratividade e usabilidade, de modo que a escolha e o tamanho da fonte foram escolhidos para garantir que o texto esteja em um tamanho confortável para leitura em dispositivos móveis, bem como a padronização de cores em tons claros parar remeter à tranquilidade e estar em harmonia com todo o aplicativo. Como explica [22], as cores estão ligadas às sensações e reflexões sensoriais e possuem grande importância para o ser humano. As cores claras psicologicamente, estar associada a emoção de calma e as sensações de paz, harmonia e leveza, afetando a parte do cérebro responsável pelo pensamento lógico e competência comunicativa.

Foram utilizados também menus e botões com ícones e descrições para que os usuários pudessem acessar os sistemas de maneira rápida e simples.

O presente protótipo apresenta primeiramente a tela inicial no qual é composta pelas principais funcionalidades do sistema conforme é apresentada na figura 2, 


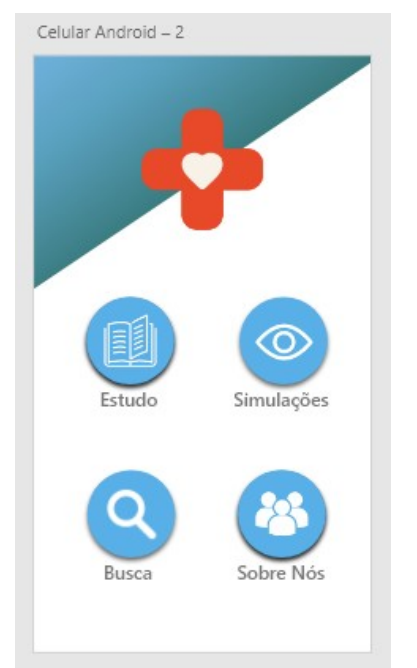

Fig. 2. Tela Inicial - Fonte: própria autora

Desde o momento que o usuário escolher a área de estudo ele será direcionado a tela onde será listado os principais assuntos referentes ao tema feridas apresentada na figura 3.

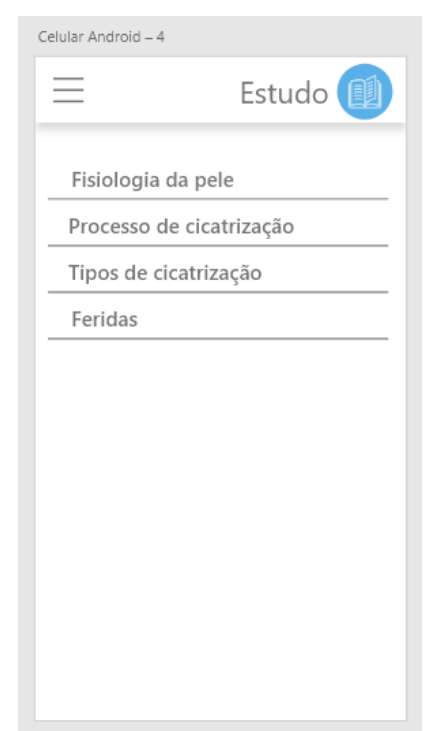

Fig. 3. Tela de listagem de estudo - Fonte: própria autora

A partir do momento que o usuário selecionar o conteúdo desejado ele será direcionado a tela de estudo que terá foco na apresentação de informações de conceitos sobre feridas. Durante a leitura textual, o usuário poderá visualizar imagens com ilustrações representativas do assunto proposto de modo que facilite a compreensão do conteúdo demonstrada na figura 4.

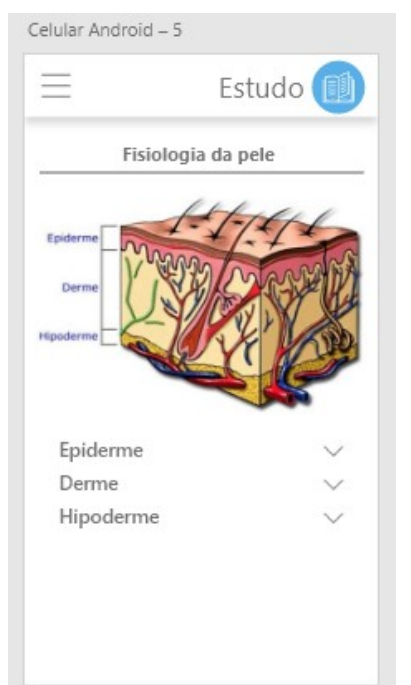

Fig. 4. Tela de estudo (Fisiologia da pele) - Fonte: própria autora

A tela de simulações conterá recursos de multimídia com o objetivo de demostrar alguma técnica ou procedimento em relação ao tema abordado, permitindo a visualização de um processo ou ação do mundo real, de modo a facilitar o entendimento de técnicas e procedimentos clínicos como pode ser observado na figura 5. Desse modo através da tela de simulações poderão ser proposta práticas passiveis de replicação no dia a dia de aprendizado do aluno.

O aplicativo também conta com a possibilidade de realizar busca para que os usuários possam procurar por um termo ou palavra relacionado aos conteúdos de feridas existente no aplicativo, como pode ser visto na figura 5.

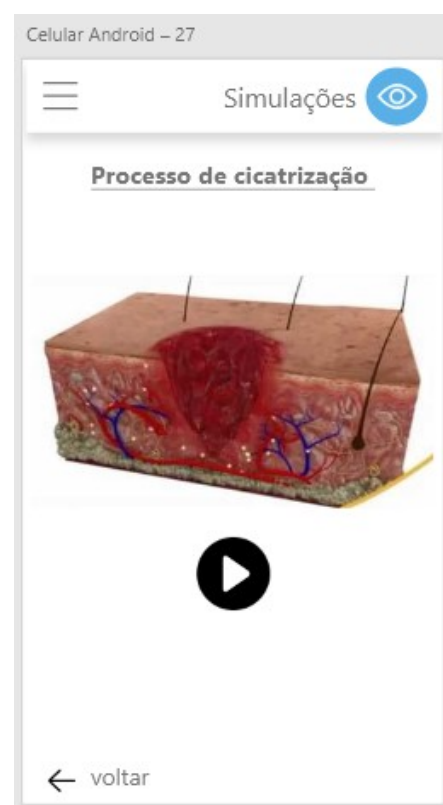

Fig. 5. Tela de simulações - Fonte: própria autora

É importante destacar que para o acesso do aplicativo proposto, não será necessário conexão com a internet ou criar perfis e contas de acesso específicos, uma vez que uma das propostas do aplicativo é que tenha uma ampla mobilidade, onde o usuário não necessite ficar preso a locais com acesso à internet para desenvolver seu ensino e aprendizagem. 
O aplicativo proposto, como já informado anteriormente, será desenvolvido para sistemas operacionais Android e IOS (Iphone OS). Conforme [23], estudos mostram que as tecnologias mencionadas, dominam mais de $90 \%$ do mercado mundial de Smartphones.

\section{CONSIDERAÇÕES FINAIS}

A tecnologia é um recurso muito rico e deve ser explorada em sala de aula, uma vez que essa ferramenta além de transformar as aulas deixando-as mais dinâmicas consegue também estar presente em praticamente todos os lugares, uma vez que as tecnologias mais utilizadas atualmente são móveis.

No cenário estudado, os resultados mostraram que, todos os professores do curso técnico em Enfermagem do IFNMG Campus Januária desejam ter uma ferramenta tecnológica que dê suporte ao ensino, e percebem a necessidade de usa-la em sala de aula.

Por meio da criação do protótipo pode perceber a importância da construção e o uso de protótipos devem fazer parte de um desenvolvimento parte do cotidiano de um game designer e de toda a equipe

A partir da criação dos protótipos pode perceber que a interface é o principal meio de comunicação entre o sistema e o usuário, nesse sentido é de extrema relevância oferecer a melhor experiência possível ao mesmo, a interface deve entregar ao usuário uma mensagem clara e objetiva a fim de atender suas necessidades sem criar dúvidas ou frustações.

As etapas percorridas durante o projeto abrem perspectivas para acreditar que o uso desse aplicativo proposto pode ser de grande eficácia no processo de ensino em enfermagem no sentido de garantir facilidade de acesso à informação independente das restrições de tempo e espaço permitindo um modo de educação mais flexível, utilizando um recurso amplamente comum no cotidiano da sociedade atual.

Como trabalho futuro será desenvolvido uma aplicação mobile fazendo a implementação dos requisitos apresentados por meio de ambiente de desenvolvimento, linguagem de programação, banco de dados, dentre outros. Visando auxiliar o ensino do tema relacionado a feridas e consequentemente contribuir na aprendizagem dos discentes com a utilização de dispositivos móveis.

\section{AGRADECIMENTOS}

Ao Instituto Federal de Educação, Ciência e Tecnologia do Norte de Minas - Campus Januária (IFNMG) pela concessão da bolsa do Programa Institucional de Iniciação Científica (PIBIC).

\section{REFERÊNCIAS}

[1] S. Hall, "HALL S . A IDENTIDADE CULTURAL NA PÓS-MODERNIDADE . 10a ED . RIO," Texto Context. - Enferm., vol. 15, no. 1, pp. 2005-2006, 2006, [Online]. Available: http:// www.scielo.br/scielo.php? script=sci_arttext\&pid=S0104-

\section{1\&lng=pt\&tlng=pt.}

[2] E. C. Lima and R. S. Appolinário, "A educação profissionalizante em enfermagem no Brasil: Desafios e perspectivas," Rev. Enferm., vol. 19, no. 2, pp. 311-316, 2011.

[3] IFNMG, "Plano de curso curso de educação profissional técnica de nível médio em enfermagem," 2014.

[4] A. C. (org) Gil, Delineamento da Pesquisa, vol. 264. 2008.

[5] A. L. P. Cogo, E. N. R. Pedro, A. P. S. S. da Silva, G. P. Valli, and A. M. Specht, "Tecnologias digitais no ensino de graduação em enfermagem: as possibilidades metodológicas por docentes," Rev. Eletrônica Enferm., vol. 13, no. 4, pp. 657-64, 2011, doi: 10.5216/ree.v13i4.12562.

[6] P. de S. Aquino, "Tecnologia Educativa no Ensino De Enfermagem Em Contracepção," Univ. Fed. Do Ceará Fac. Farmácia, Odontol. e Enferm. Programa pós-Graduação em Enferm., p. 102, 2010, [Online]. Available: http://www.repositorio.ufc.br/bitstream/riufc/2140/1/2010_tese_psaquino.pdf.

[7] UNESCO, Diretrizes de politicas para a aprendizagem móvel. 2014.

[8] A. M. C. Moura, "Apropriação do telemóvel como ferramenta de mediação em mobile learning. Estudo de caso em contexto educativo," p. 630, 2010.

[9] C. R. Y. Mashuda, J. O. Cazzetta, W. L. Berto, and O. Godoy, "M-Learning: a Utilização de Dispositivos Móveis no Contexto Educacional," UNOPAR Científica Ciências Exatas e Tecnológicas, vol. 9, no. 1, pp. 61-66, 2010, [Online]. Available: http:// revista.pgsskroton.com.br/index.php/exatas/article/view/597/567.

[10] M. S. Bhullar, "A new method of learning: MLearning (Mobile Learning)," Proc. 9th Int. Conf. Comput. Sci. Educ. ICCCSE 2014, no. Iccse, pp. 322-325, 2014, doi: 10.1109/ ICCSE.2014.6926478.

[11] M. A. Mulyani, A. Razzaq, W. H. Sumardi, and M. Anshari, "Smartphone Adoption in Mobile Learning Scenario," Proc. 2019 Int. Conf. Inf. Manag. Technol. ICIMTech 2019, vol. 1, no. August, pp. 208-211, 2019, doi: 10.1109/ ICIMTech.2019.8843755.

[12] P. Campanella, "Mobile learning: New forms of education," ICETA 2012 - 10th IEEE Int. Conf. Emerg. eLearning Technol. Appl. Proc., pp. 5156, 2012, doi: 10.1109/ICETA.2012.6418282.

[13] R. Picek and M. Grcic, "Evaluation of the potential use of m-learning in higher education," Proc. Int. Conf. Inf. Technol. Interfaces, ITI, pp. 63-68, 2013, doi: 10.2498/iti.2013.0583.

[14] M. Marconi and E. Lakatos, Fundamentos de metodologia cientifica. 2003.

[15] F. CHIEN, "Estudo comparativo de técnicas para 
levantamento de requisitos de aplicativos móveis," 2018.

[16] A. K. O. Sato, "Game Design e Prototipagem: Conceitos e Aplicações ao Longo do Processo Projetual," Proc. SBGames 2010, pp. 74-84, 2010.

[17] J. Nielsen, "Enhancing the explanatory power of usability heuristics," p. 210, 1994, doi: 10.1145/259963.260333.

[18] C. Maciel, J. L. T. Nogueira, and L. N. Ciuffo, "Avaliação Heuristica de Sítios na Web Avaliação Heurística de Sítios na Web,” no. January, 2015.

[19] C. M. V. Costa, I. Chagas, J. M. Carvalho, and W. E. P. -, "Diretrizes Clínicas Protocolos Clínicos 035 Feridas Hospitalares II," p. 252, 2013, [Online]. Available: www.fhemig.mg.gov.br.

[20] A. M. Ferreira, M. A. Rigotti, S. B. Pena, D. da S. Paula, I. B. Ramos, and V. D. M. Sasaki, "Conhecimento e prática de acadêmicos de enfermagem sobre cuidados com portadores de feridas," Esc. Anna Nery, vol. 17, no. 2, pp. 211-219, 2013, doi: 10.1590/s1414-81452013000200002.

[21] G. F. da C. Morais, S. H. dos S. Oliveira, and M. J. G. O. Soares, "Avaliação de feridas pelos enfermeiros de instituições hospitalares da rede pública," Texto Context. - Enferm., vol. 17, no. 1, pp. 98-105, 2008, doi: 10.1590/s010407072008000100011.

[22] P. V. G. de Azevedo, J. Valentim, and P. S. Queiroz, "ESTUDO SOBRE O EFEITO EMOCIONAL DAS CORES NA CONSTRUÇÃO DE UM APLICATIVO PARA AUXILIAR EDUCANDOS, 2019.

[23] L. R. Queiroz, "IPhone, Android, e a consolidação da cultura do smartphone: o papel do IPhone e do Sistema Operacional Android como catalisadores da consolidação no mercado de smartphones em escala global," Rev. Tecnol. e Soc., vol. 14, no. 30, pp. 47-70, 2018, doi: 10.3895/rts.v14n30.5413. 


\section{ANEXO}

\section{ELABORAÇÃO DE UM SOFTWARE EDUCACIONAL PARA AUXÍLIO DO PROCESSO DE ENSINO NA ENFER-}

MAGEM

Este questionário integra o Trabalho de Conclusão de Curso de Tecnologia de Análise e Desenvolvimento de Sistemas do IFNMG - Campus Januária. O objetivo é identificar as dificuldades enfrentadas pelos Professores do Curso Técnico em Enfermagem do IFNMG - Campus Januária no ensino dos conteúdos das disciplinas Fundamentos de Enfermagem.

1- Dentre os conteúdos apresentados na disciplina Fundamentos de Enfermagem I, em sua opinião qual apresenta maior limitação na prática de ensinar?

( ) Higienização das mãos

( ) Colocação e retirada de luvas de procedimento / luvas estéreis

( ) Aferição de sinais vitais

( ) Dor e manejo de alterações,

( ) Aferição de dados antropométricos (adultos)

( ) Glicemia capilar / curva glicêmica

( ) Crioterapia / termoterapia

( ) Arrumação de leito

( ) Posições para exames

( ) Transporte / mobilização / imobilização do paciente

( ) Restrição do paciente

( ) Higiene (corporal, oral, íntima, auricular, nasal, capilar, corte de unhas)

( ) Troca de fraldas/ uso de comadre e marreco

( ) Troca de bolsa de ostomia

2- Dentre os conteúdos apresentados na disciplina Fundamentos de Enfermagem II, em sua opinião qual apresenta maior limitação na prática de ensinar?

( ) Nebulização / inalação

( ) Oxigenioterapia

( ) Punção venosa

( ) Administração de medicamentos (tópico, oral, nasal, auricular, oftalmológico, parenteral, vaginal e retal)

( ) Enema / enteróclise

( ) Cálculo de medicação

( ) Tratamento de feridas

( ) Retirada de pontos

3- Dentre os conteúdos apresentados na disciplina Fundamentos de Enfermagem III, em sua opinião qual apresenta maior limitação na prática de ensinar?

( ) Coleta de materiais para exame

( ) Eletrocardiograma (ECG)

( ) Hemotransfusão

( ) Cuidados com traqueostomia

( ) Aspiração de secreções

( ) Sondagem gastroenteral

( ) Sondagem vesical (alívio / demora)

( ) Reno de tórax, cuidados com drenagem torácica

( ) Preparo do corpo pós-morte. 
4- Em sua opinião, o desenvolvimento de um aplicativo auxiliaria no ensino dos conteúdos ministrados nas disciplinas Fundamentos I, II e III.

( ) SIM

( ) NÃO

( ) TALVEZ

5- Em sua opinião atividades interativas como jogos, animações e vídeos poderia auxiliar a prática de ensino em sala de aula?

( ) SIM

( ) NÃO

( ) TALVEZ 